Research Article

\title{
A SIMULATION MODEL FOR CUSTOMER FLOW ANALYSIS IN A COMMERCIAL BANK IN NIGERIA
}

\author{
Mayowa Rafiat AJIBOYE ${ }^{I^{*}}$, Isiaka ISMAIL ${ }^{2}$, Temitope Olubanjo KEHINDE ${ }^{3}$, Adewale ABE ${ }^{4}$
}

In view of competitiveness and increasing search for improved services in Nigerian commercial banks, there are some organizational changes necessary to facilitate this fate. Recently, the possibility of applying operational research techniques, more specifically computer simulation, was raised to address the problem of customer queues at bank branches. No bank has been punished with a fine if there are customers waiting for more than a certain period of time in the queues to be served. The solution to the problem sought to offer customer-care to the crowd in all bank branches in Nigeria. In view of this, the present work proposes a computer simulation model to study the flow of customers in a branch of the bank. This model simulates service capacity and time in the face of various hypothetical scenarios to which a bank branch may be subject using the Rockwell Automation Arena ${ }^{\circledR}$ v15 software. The results make it possible to evaluate new policies for increasing the quality of service and compliance with easy customer-care operational service. The generated scenarios were evidence alternatives that would reduce waiting times with only a few minor alterations. Thereby allowing the service with maximum waiting time the standards required.

Key words: Simulation, commercial-banks, queuing, Arena, customer-care

\section{Introduction}

Waiting lines have always characterized a problem at commercial banks. Initially, parallel lines were used, one for each service desk. Subsequently, these parallel lines were replaced by a single line, where all customers wait in a single line for the first available window. Currently, in addition to the single line, bank branches provide customers with a wide variety of alternative services and in an automated way, either at branches or over the internet, but they have not, however, been able to eliminate the long lines inside the bank branches.

With the emergence of new products and services offered by the banking sector, banking institutions, aware of their responsibilities, have invested significantly in actions aimed at offering personalized and high-quality service to all their customers and users.

\footnotetext{
${ }^{1}$ Department of Accounting, Al-Hikmah University, Ilorin, Nigeria (rafiat.ajiboye@yahoo.com) Dhttps://orcid.org/0000-000301777-4799

2 Department of Electrical \& Electronic Engineering, Ekiti State University, Ado-Ekiti, Nigeria, (isiaka.ismail@eksu.edu.ng) https://orcid.org/0000-0003-2428-2383

3 Department of Industrial \& Production Engineering, University of Ibadan, Ibadan, Nigeria, (kehindeolubanjo@gmail.com) https://orcid.org/0000-0001-7703-7395

${ }^{4}$ Department of Electrical \& Electronic Engineering, Ekiti State University, Ado-Ekiti, Nigeria, (adewale.abe@eksu.edu.ng) https://orcid.org/0000-0002-3285-1833
} 
Due to modernization and increasing population, the number of people served by banks grow daily and the services offered are increasing in numbers. To further aggravate this problem, there is a seasonality inherent in banking operations, such as payday for civil servants, the eve of holidays, end and beginning of the month, lunchtime and close to the closing of the branch, etc.

These issues generate changes in the dynamics of the client flow and make it difficult to predict resources allocation and, consequently, control the time of the agency's operations. It is often common for a customer to wait for more than 30 minutes to be served or perform an operation at an ATM. This is a typical problem and is repeated in many agencies in the country. Following the worldwide trend of expanding electronic service as a cost reducer, the banking sector has been gradually expanding investment in electronic equipment to increase the range of services, facilitate the service and convenience of its customers.

According to Abensur [1], the need for the banking network to reduce administrative and operational costs without prejudice to the quality of service has raised the segment to greater investment. New technologies such as ATMs allow banks to offer their customers service channels such as withdrawals, deposits, bill payments, transfers, and investments.

However, many customers still prefer traditional service, directly at the counter, as they believe that personal contact is essential. Therefore, offering a quality service means adjusting consumer expectations on a consistent basis, that is, if the perceived service reaches or exceeds the expected service, customers feel able to use the service provider again [2-4]. The search for process improvement is an important factor for the institutional success of any organization, regardless of its size, whether public or private, as long as it is carried out in a structured way and is understood by everyone in the organization. Therefore, if, on the one hand, there is an increase in technology to reduce the queues in the attendance at the counters, and on the other hand, there is a reduction in the number of attendants, the problem will persist if there are still queues at conventional ATMs.

Recently, this problem was studied by Favaretto and proposed the approach from the perspective of operational research [5]. He identified the complexity inherent to the dynamics of customer flow in bank branches and characterized it as a typical problem to be addressed by computer simulation. In these works, it was concluded, among other things, that considering the usual costs of banking operations, the stipulated time is compatible with the amount of the fine adopted, forcing the bank to operate within the law. In the work of Paradi and Zhu, basic procedures were presented for data sampling and the construction of a simulation model for a bank branch [6].

In view of this, it becomes necessary to build a model that allows studying the performance of the process under certain conditions, allowing the observation of behaviour and enabling the comparison of different scenarios. Thus, the present work aimed to create a computer simulation model from data collected directly at a bank branch to assess the flow of customers in critical periods of operation. From the developed model, several hypotheses were presented that could minimize or even solve the issue. The scenarios were simulated according to different situations that the agency could be subject to at different times of operation.

\section{Description of the Bank Branch}

The $\mathrm{X}$ branch chosen for this study has the services of manual cashiers (conventional service) and ATMs (automatic teller machines or self-service) with independent single lines, thus characterizing it as a typical agency with the services under study. At the request of management, the agency was not identified. 
The branch has two floors, as shown in Figures 1 and 2. As seen in Figure 1, the lower floor, the entrance to the branch and right after the ATM windows. After the revolving door are the information and general assistance desks, where customers take their passwords and head for the stairs. On the lower floor, there are also seven tables for managers. There are seven ATMs for self-service (two of which were inactive). Two employees assist customers with difficulties in operating the machines. Next to the stairs, there are two information and general assistance windows, where the customer also takes the password for assistance from the manual boxes on the upper floor.
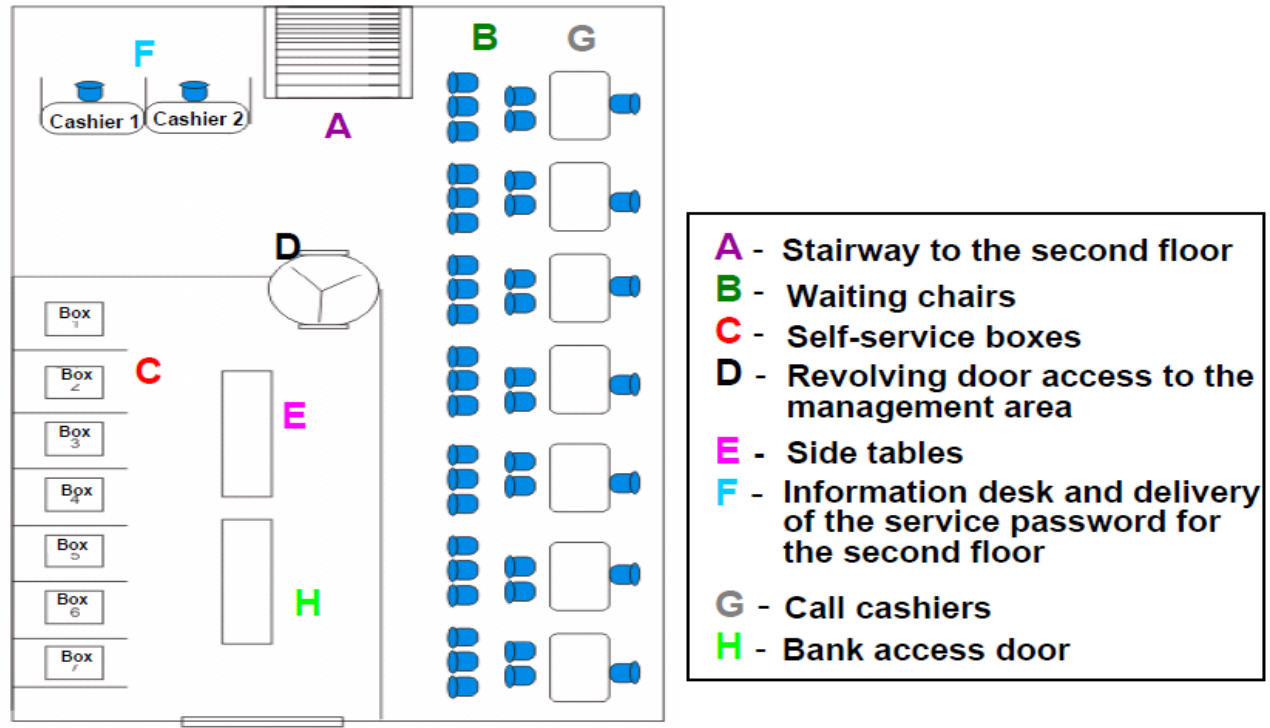

Figure 1. Ground floor of the Agency.

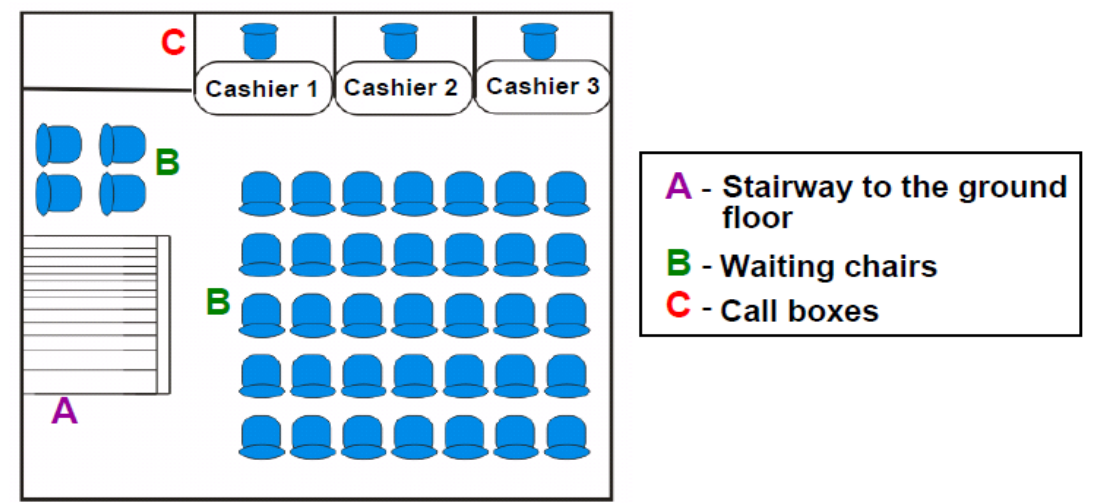

Figure 2. Top floor of the Agency.

On the upper floor, shown in Figure 2, there are manual assistance windows and chairs for waiting for assistance. Of these windows, two operated regularly and one was inactive. One attendant is designed specifically for conventional customers and the other is designed to serve preferred customers (elderly, pregnant women, etc.). If this type of customer is not on hold, he also serves conventional service customers.

At the agency, the queuing and password system, that is, the customer arrives, picks up the password, waits on the chairs destined for that purpose, and is attended to by the order of arrival.

The service points are:

a. Manual assistance: 2 boxes, open from 10 am to $4 \mathrm{pm}$.

b. Self-service: 5 ATMs, open from 8 am to $8 \mathrm{pm}$. 
During the observation of the system, three types of assistance were identified:
i. $\quad$ Customer service execution - preferential (manual service);
ii. Customer service execution - conventional (manual service);
iii. Customer self-service (ATM service).

\section{Description of the Simulation Model}

For the development of the model presented in this work, the methodology was proposed by [7, 8], composed of problem formulation and analysis; project planning; formulation of the conceptual model; macro-information and data collection; model translation; verification and validation; experimental design; comparison of systems and identification of the best solutions; documentation and presentation of results and implementation. The verification and validation were carried out also using the methodology proposed by Sargent $[9,10]$. The conceptual model was developed using the logical elements proposed by Montevechi, et al. [11].

The translation of the conceptual model of the system to the computer simulation model was performed using the Rockwell Automation Arena ${ }^{\circledR}$ v15 software. The data were collected between September 1 and September 30, 2019, as they are typical days of high demand in bank branches and from this, it was possible to observe that of the 4523 customers who used conventional cash, $82 \%$ were of normal service and $18 \%$ were in priority service.

Two people with stopwatches were positioned in strategic places for data collection. One person was located next to the ATMs and the other to the executive ATMs, collecting customer arrival times independently. On the second day, service times were collected, where, once again, one person was located next to the ATMs and the other one next to the executive ATMs.

Figures 3 and 4 show the arrival times of customers at the agency for executive and automatic assistance, throughout the working day (10 am to $4 \mathrm{pm}$ ) and at 15 minute intervals.

It can be seen in these graphs that the behaviour of the flow of customers arriving at the bank branch is very irregular, presenting strong fluctuations, sharply and throughout the period. In this way, the difficulty of finding a possible distribution of probabilities that adequately represents the stochastic behaviour of the variables under analysis is characterized. Thus, it was decided to insert this data into the computational model through resources for generating shifts, the Schedule. This feature establishes rules that will be in effect for certain periods of time. Thus, it is possible to generate values very close to those presented in the respective Figures 3 and 4.

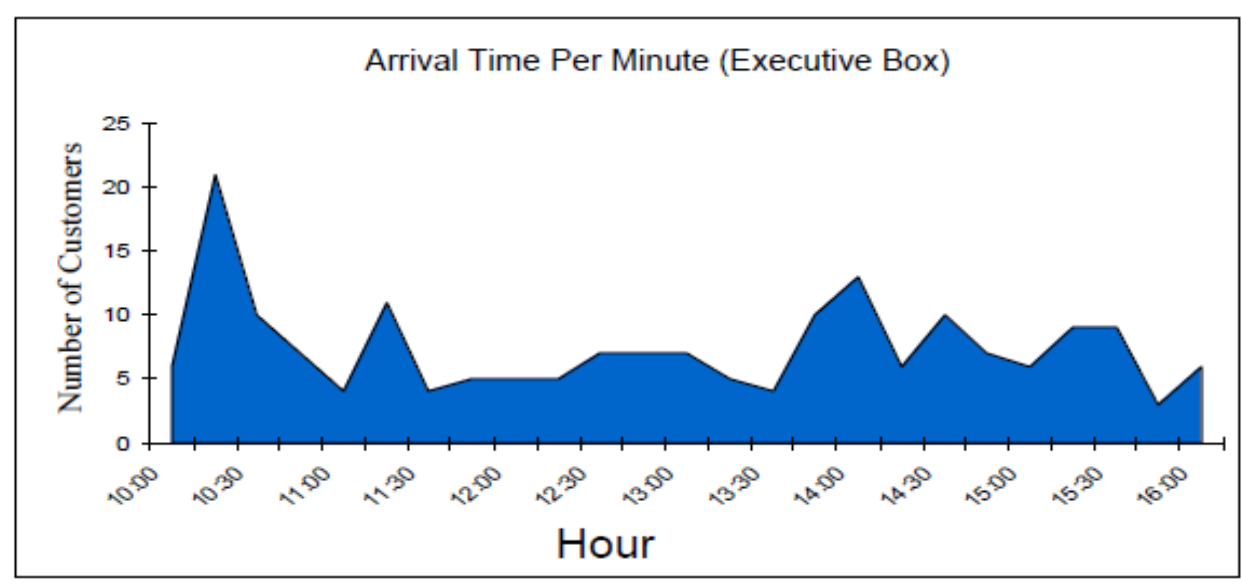

Figure 3. Customer arrival times per minute for manual service. 


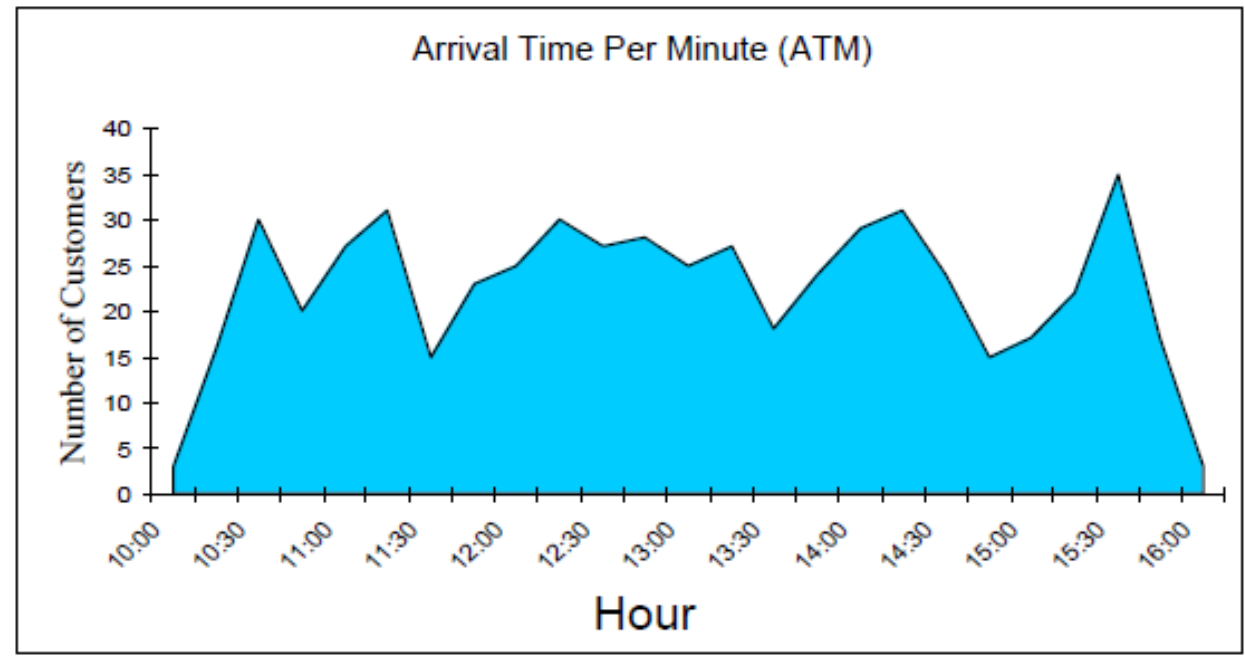

Figure 4. Customer arrival times per minute for automated service.

Regarding service times, the behaviour was more homogeneous, oscillating more smoothly around an average. This result was already expected, as it is an operation carried out in a more regular and systematic way, by one person, who performs the service. These data were processed by the Input Analyzer software, complementary to Rockwell Automation Arena ${ }^{\circledR}$ v15 software, to determine the best probability distribution that adheres to the set of values. The elements $F_{2}, F_{3}$ and $F_{4}$, in the legend of Figure 5, show the theoretical distributions of probabilities that best adhere to the stochastic behaviour of the variables.

The main purpose of the Input Analyzer software is to identify the best theoretical probability distribution, using the following adherence tests, namely: Chi-square and K-S [12].

After collecting data and identifying the behaviour of the variables that represent the dynamics of the customer flow at the bank branch, the conceptual model of the system was then shown, shown in Figure 5.

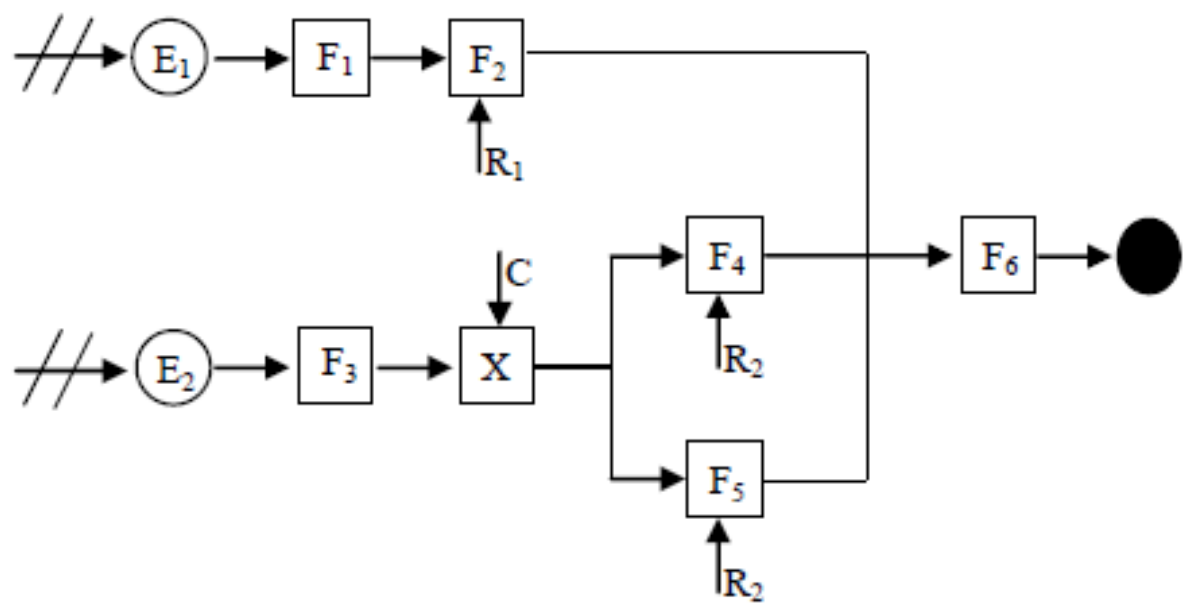

Figure 5. Conceptual model of customer flow at the bank branch.

The description of the parameters used in the banking service operation are stated in Table 1 below. Two Arena ${ }^{\circledR}$ v15 schedule modules (referring: $\mathrm{E}_{1}$ and $\mathrm{E}_{2}$, in Figure 5) were used to input data regarding the arrival of customers who were destined for ATMs and executive cashiers. 
Table 1. Banking service operation parameters.

\begin{tabular}{lll}
\hline & \multicolumn{1}{c}{ Description } & \multicolumn{1}{c}{ Parameter } \\
\hline $\mathrm{E}_{1}$ & Arrival of Customers for Automatic Service & Schedule - referring to Figure 4 (one customer at a time) \\
$\mathrm{E}_{2}$ & Arrival of Customers for Manual Service & Schedule - referring to Figure 3 (one customer at a time) \\
$\mathrm{F}_{1}$ & Automatic Answering Queue Location & Infinite capacity \\
$\mathrm{F}_{2}$ & Auto Answer & Normal log (average: 0.0725 and deviation: 0.0656) min \\
$\mathrm{F}_{3}$ & Manual Service Queue Location & Infinite capacity \\
$\mathrm{F}_{4}$ & Conventional Manual Service & 0.5 + Normal log (average: 3.1 and deviation: 2.76$)$ min \\
$\mathrm{F}_{5}$ & Manual Attendance Preference & 0.5 + Normal log (average: 3.1 and deviation: 2.76$)$ min \\
$\mathrm{F}_{6}$ & Exit Location & Infinite capacity \\
$\mathrm{R}_{1}$ & Electronic Cashier & 5 Answering Machines \\
$\mathrm{R}_{2}$ & Execution Cashier & 3 Service windows \\
$\mathrm{C}$ & Preferential Service Control & 15\% of Preferred Customers \\
\hline
\end{tabular}

${ }^{1}$ If there is no preferred customer, the first in line is served. .

This procedure was adopted, because although all clients use the same access door when the agency arrives, the behaviour of the two services is different, justifying the collection made separately.

The customer who goes to the ATM, after performing self-service, leaves the system. The customer who goes to the cashier after his arrival is characterized by his type of service, whether preferential or conventional. If the customer has a preferential service, he goes to his specific cashier and is served. In the same way, the conventional customer is addressed to the conventional execution cashier and is served, if the customer of this service is in the queue and the preferred execution cashier is idle, the conventional customer goes to the preferred cashier to be served. Then, after the service, this type of customer leaves the system.

After the translation of the conceptual model to the simulation model in Arena ${ }^{\circledR}$ v15, verification, and validation were performed, following the methodology proposed by Sargent [9, 10]. In this stage, the model was monitored step by step, that is, all incoming customers were accompanied to their service and checked out. It was also found that the results obtained matched those of the real system, through an agency employee.

\section{Analysis and Discussion of Results}

Six hypothetical scenarios were designed, in order to allow the assessment of different situations in relation to the flow of clients that the agency maybe subject to, in typical situations of its daily life. These scenarios are described below and consider both a situation of increase with a reduction in the arrival rate, as well as an increase in resources.

The reduction in the arrival rate makes it possible to evaluate days with a less intense flow, characteristic of the system's seasonality.

a. Scenario 1 - Increasing the capacity of the manual gearbox from one to two, in addition to the preferred gearbox.

b. Scenario $2-10 \%$ increase in the number of customers arriving at the agency.

c. Scenario 3 - Increase of $20 \%$ in the number of customers arriving at the agency.

d. Scenario 4 - Increase in the number of preferred customers from $15 \%$ to $25 \%$.

e. Scenario 5 - Increase in the number of ATMs from 5 to 6 resources.

f. Scenario $6-50 \%$ reduction in the number of customers arriving at the agency.

50 replications were used in the simulations of the respective scenarios. In the analysis of these scenarios, the average and the maximum number of customers waiting in the queues, the average and the maximum waiting times in the queues and the average and maximum occupancy rates of the service resources were considered. The results of the simulations of the respective scenarios are presented in

Tables 2 to 7. 


\section{Scenerio 1:}

The increase in the number of conventional execution boxes from one to two boxes was simulated. The results show only the rates and times that have changed.

Table 2. Represention of results for scenario 1.

\begin{tabular}{|c|c|c|c|c|}
\hline & \multicolumn{2}{|c|}{ Actual situation } & \multicolumn{2}{|c|}{ Scenerio 1} \\
\hline & Medium & Maximum & Medium & Maximum \\
\hline \multicolumn{5}{|l|}{ CONVENTIONAL Execution Box } \\
\hline Number of customers in the queue & 7 & 34 & 2 & 22 \\
\hline Waiting time & $15.45 \mathrm{~min}$ & $78.13 \mathrm{~min}$ & $2.87 \mathrm{~min}$ & $33.31 \mathrm{~min}$ \\
\hline Cashier occupancy rate & $92.62 \%$ & $99.99 \%$ & $66.30 \%$ & $83.83 \%$ \\
\hline \multicolumn{5}{|l|}{ PREFERENCIAL Execution Box } \\
\hline Number of customers in the queue & 1 & 6 & 1 & 9 \\
\hline Waiting time & $3.84 \mathrm{~min}$ & $51.09 \mathrm{~min}$ & $2.41 \mathrm{~min}$ & $47.17 \mathrm{~min}$ \\
\hline Cashier occupancy rate & $81.72 \%$ & $99.93 \%$ & $44.64 \%$ & $66.44 \%$ \\
\hline
\end{tabular}

\section{Results and observations:}

From the results obtained in the simulation of this scenario, it was possible to observe that the number of customers in the conventional executive cashier queue, service time and cash occupancy rate decreased. And in the preferred cash register, despite having undergone changes, its impact was minimal. However, in both situations in scenario 1, the law was sometimes not enforced. However, the occupancy rate of the conventional cashier resource went from $99.9 \%$ to $83.8 \%$ in maximum situations. Thus, moving to a more adequate occupancy rate for a human being.

\section{Scenerio 2:}

A proportional increase in the data collected for the agency arrival rate by $10 \%$ was simulated. The arrival rate increased from 738 to 801 customers, maintaining the same behavior of the data collected for both arrivals.

Table 3. Represention of results for scenario 2.

\begin{tabular}{|c|c|c|c|c|}
\hline & \multicolumn{2}{|c|}{ Actual situation } & \multicolumn{2}{|c|}{ Scenerio 2} \\
\hline & Medium & Maximum & Medium & Maximum \\
\hline \multicolumn{5}{|c|}{ CONVENTIONAL Execution Cashier } \\
\hline Number of customers in the queue & 7 & 34 & 11 & 49 \\
\hline Waiting time & $15.47 \mathrm{~min}$ & $78.11 \mathrm{~min}$ & $21.64 \mathrm{~min}$ & $99.16 \mathrm{~min}$ \\
\hline Cashier occupancy rate & $92.62 \%$ & $99.99 \%$ & $95.60 \%$ & $100 \%$ \\
\hline \multicolumn{5}{|l|}{ PREFERENCIAL Execution Cashier } \\
\hline Number of customers in the queue & 1 & 6 & 1 & 9 \\
\hline Waiting time & $3.84 \mathrm{~min}$ & $51.09 \mathrm{~min}$ & $4.23 \mathrm{~min}$ & $45.34 \mathrm{~min}$ \\
\hline Cashier occupancy rate & $92.62 \%$ & $99.93 \%$ & $89.30 \%$ & $99.90 \%$ \\
\hline \multicolumn{5}{|l|}{ ELECTRONIC Cashier } \\
\hline Number of customers in the queue & 3 & 36 & 5 & 45 \\
\hline Waiting time & $1.31 \mathrm{~min}$ & $16.90 \mathrm{~min}$ & $2.40 \mathrm{~min}$ & $23.33 \mathrm{~min}$ \\
\hline Cashier occupancy rate & $76.56 \%$ & $85.22 \%$ & $83.92 \%$ & $94.17 \%$ \\
\hline
\end{tabular}

\section{Results and observations:}

It was observed that in the conventional executive cashier, the number of customers in the queue, the service time and the cash occupancy rate increased a lot. In the preferred executive cash register, the increase was smaller. At the ATM, the impact was great, and there was an increase in all items.

\section{Scenerio 3:}

In this scenario, the number of customers who arrived was 858 , due to the $20 \%$ rate increase. 
Table 4. Represention of results for scenario 3.

\begin{tabular}{|c|c|c|c|c|}
\hline & \multicolumn{2}{|c|}{ Actual situation } & \multicolumn{2}{|c|}{ Scenerio 3} \\
\hline & Medium & Maximum & Medium & Maximum \\
\hline \multicolumn{5}{|c|}{ CONVENTIONAL Execution Cashier } \\
\hline Number of customers in the queue & 7 & 34 & 16 & 75 \\
\hline Waiting time & $15.46 \mathrm{~min}$ & $78.12 \mathrm{~min}$ & $31.51 \mathrm{~min}$ & $120.70 \mathrm{~min}$ \\
\hline Cashier occupancy rate & $92.62 \%$ & $99.99 \%$ & $98.08 \%$ & $100 \%$ \\
\hline \multicolumn{5}{|c|}{ PREFERENCIAL Execution Cashier } \\
\hline Number of customers in the queue & 1 & 6 & 2 & 9 \\
\hline Waiting time & $3.83 \mathrm{~min}$ & $51.08 \mathrm{~min}$ & $4.65 \mathrm{~min}$ & $50.05 \mathrm{~min}$ \\
\hline Cashier occupancy rate & $81.72 \%$ & $99.93 \%$ & $95.12 \%$ & $100 \%$ \\
\hline \multicolumn{5}{|l|}{ ELECTRONIC Cashier } \\
\hline Number of customers in the queue & 3 & 36 & 11 & 82 \\
\hline Waiting time & $1.30 \mathrm{~min}$ & $16.91 \mathrm{~min}$ & $5.60 \mathrm{~min}$ & $40.26 \mathrm{~min}$ \\
\hline Cashier occupancy rate & $76.56 \%$ & $85.22 \%$ & $91.43 \%$ & $96.10 \%$ \\
\hline
\end{tabular}

\section{Results and observations:}

As in the previous scenario, the queues increased, but with greater intensity.

\section{Scenerio 4:}

This scenario was simulated with the same rate of arrival of customers at the branch, with the percentage of preferred customers increasing from $15 \%$ to $25 \%$.

Table 5. Represention of results for scenario 4.

\begin{tabular}{|c|c|c|c|c|}
\hline & \multicolumn{2}{|c|}{ Actual situation } & \multicolumn{2}{|c|}{ Scenerio 4} \\
\hline & Medium & Maximum & Medium & Maximum \\
\hline \multicolumn{5}{|c|}{ CONVENTIONAL Execution Cashier } \\
\hline Number of customers in the queue & 7 & 34 & 6 & 34 \\
\hline Waiting time & $15.46 \mathrm{~min}$ & $78.12 \min$ & $14.76 \mathrm{~min}$ & $83.36 \mathrm{~min}$ \\
\hline Cashier occupancy rate & $92.62 \%$ & $99.99 \%$ & $89.61 \%$ & $99.98 \%$ \\
\hline \multicolumn{5}{|c|}{ PREFERENCIAL Execution Cashier } \\
\hline Number of customers in the queue & 1 & 6 & 2 & 14 \\
\hline Waiting time & $3.83 \mathrm{~min}$ & $51.08 \mathrm{~min}$ & $5.36 \mathrm{~min}$ & $60.99 \min$ \\
\hline Cashier occupancy rate & $81.72 \%$ & $99.93 \%$ & $85.06 \%$ & $99.93 \%$ \\
\hline
\end{tabular}

\section{Results and observations:}

In this scenario, the preferred executive cash register had an increase in all criteria, whereas in the conventional executive cash register there was a decrease in the number of customers in the queue, in the service time and occupancy rate, but not enough to comply with the law.

\section{Scenerio 5:}

In this scenario, the behavior of the system was analyzed by increasing the number of ATMs, from 5 to 6 machines.

Table 6. Represention of results for scenario 5 .

\begin{tabular}{lcccc}
\hline & \multicolumn{2}{c}{ Actual situation } & \multicolumn{2}{c}{ Scenerio 4 } \\
\cline { 2 - 5 } ELECTRONIC Cashier & Medium & Maximum & Medium & Maximum \\
\hline Number of customers in the queue & 3 & & & 1 \\
\hline Waiting time & $1.31 \mathrm{~min}$ & $16.90 \mathrm{~min}$ & $0.39 \mathrm{~min}$ & $10.26 \mathrm{~min}$ \\
\hline Cashier occupancy rate & $76.56 \%$ & $85.22 \%$ & $64.06 \%$ & $73.89 \%$ \\
\hline
\end{tabular}

\section{Results and observations:}

In this scenario, it was noticed that there was a drop in all the evaluated points, due to the increase in the resource's capacity. 


\section{Scenerio 6:}

In the last simulated scenario, the situation with which the agency spends during non-peak days was analyzed, so a $50 \%$ drop in arrival rate was estimated. Soon it was possible to analyze what the agency's idleness these days. The number of clients who arrived at the agency to simulate this scenario was 372 .

Table 7. Represention of results for scenario 6.

\begin{tabular}{|c|c|c|c|c|}
\hline & \multicolumn{2}{|c|}{ Actual situation } & \multicolumn{2}{|c|}{ Scenerio 6} \\
\hline & Medium & Maximum & Medium & Maximum \\
\hline \multicolumn{5}{|c|}{ CONVENTIONAL Execution Cashier } \\
\hline Number of customers in the queue & 7 & 34 & 1 & 18 \\
\hline Waiting time & $15.46 \mathrm{~min}$ & $78.12 \mathrm{~min}$ & $4.20 \mathrm{~min}$ & $52.19 \mathrm{~min}$ \\
\hline Cashier occupancy rate & $92.62 \%$ & $99.99 \%$ & $63.82 \%$ & $83.47 \%$ \\
\hline \multicolumn{5}{|l|}{ PREFERENCIAL Execution Cashier } \\
\hline Number of customers in the queue & 1 & 6 & 0 & 5 \\
\hline Waiting time & $3.83 \mathrm{~min}$ & $51.08 \mathrm{~min}$ & $1.23 \mathrm{~min}$ & $30.43 \mathrm{~min}$ \\
\hline Cashier occupancy rate & $81.72 \%$ & $99.93 \%$ & $26.37 \%$ & $49.05 \%$ \\
\hline \multicolumn{5}{|l|}{ ELECTRONIC Cashier } \\
\hline Number of customers in the queue & 3 & 36 & 0 & 5 \\
\hline Waiting time & $1.30 \mathrm{~min}$ & $16.91 \mathrm{~min}$ & $0.01 \mathrm{~min}$ & $2.62 \mathrm{~min}$ \\
\hline Cashier occupancy rate & $76.56 \%$ & $85.22 \%$ & $31.95 \%$ & $38.18 \%$ \\
\hline
\end{tabular}

\section{Results and observations:}

In this analysis the expected occurred. On normal days, the agency's resource capacity met the needs of the demand, but in the waiting time for conventional and preferred cash, they still exceeded the limit determined by law in some moments of the simulations.

\section{Analysis of Scenario}

In scenario 1, when there was an increase in the resource capacity of conventional executive cash, the flow of clients improved considerably at the branch, through the reduction of queues and service time. Through this result, the agency is practically complying with the law, but in the preferred cashier the waiting time is still long, so a fourth cashier is needed during peak hours. The occupancy rate also decreased, giving the agency's employee time off to provide better quality care.

In scenario 2, the $10 \%$ increase in the arrival rate influenced the increase in the number of customers who arrived at the branch, so the number of customers in the queues and the occupancy rate also increased in all cashiers, but not significantly, due to these already high. However, in scenario 3, with the $20 \%$ increase, this impact was greater, as the number of customers increased significantly and the cashiers had their occupancy rate of $100 \%$.

In scenario 4, with the increase in the percentage of preferred customers, the cash flow of this type of service was overloaded, but the load of the conventional service box decreased.

In scenario 5, the number of ATMs was increased from 5 to 6 counters, in this situation the machine occupancy rate decreased even though it was not necessary, since the machines can have their occupancy rate up to $100 \%$. But due to the arrival rate being very dispersed, in some moments small and in others very large, the addition of this window reduced the queues for this service, which can be seen in Figure 3, providing more satisfaction to the customer in self-service.

In scenario 6 , the customer arrival rate decreased by $50 \%$, to analyze what the agency's situation would be like on non-peak days. For this situation, the capacity of the agency's current resources meets its needs. 
The analyzed scenarios can be divided into two classes. First, the scenarios that propose an improvement in the current situation of the agency, such as, for example, the increased capacity for appeal and, later, the scenarios that simulate situations that may occur to worsen the current situation of the agency, such as the increase in the rate of arrival of customers.

However, as observed in the analysis of the scenarios, on peak days changes are needed in the real situation of the agency, so that it is complying with the "queue law" and providing more quality in the service of its clients.

Through the comparisons made between the scenarios presented, those that improved the service conditions at the agency, represented in scenarios 4, 5 and 6, stood out. From these comparisons, it was possible to analyze how the average service time would be, the average number of queued customers and the average occupancy rate according to the best scenarios as proposed by the agency.

\section{Conclusions}

The model adequately described the behavior of the system under study and proved to be an important tool to assist bank branch managers in making decisions about the operation of resources and to better control the behavior of queues. From the computer simulations, it was possible to evaluate that, in peak days, there is a need to increase the capacity of resources in the studied agency, so that it can be fulfilling the requirements determined by the "queuing law".

From the initial moment of data collection, it was possible to observe that the occupancy rate of the agency's employees was high and, from the results of the simulations, it was possible to verify that the employees had their occupancy rate beyond normal. If the rate of arrival of customers increases further, as analyzed in some scenarios, it was found that the attendants, in manual cashiers would further increase their respective occupancy rates to extremely high rates, compromising the performance of functions.

It is also worth mentioning the application of Integrated DEFinition Methods of simulation (IDEF-SIM) in conceptual modeling. As indicated in the article by Montevechi, et al. [11], the creation of the conceptual model, using appropriate syntax and semantics, makes the computational model translation stage more agile, improving the development of the simulation project as a whole.

\section{References}

[1] Abensur, E.O. (2011). Banking operations using queuing theory and genetic algorithms," Produto \& Produção, 12(2), 69 - 86.

[2] Kelton W., Sadowski, R., Sturrock, D.T. Simulation with Arena, $4^{\text {th }}$ Edition, McGraw-Hill, New York, USA, 2007.

[3] Cardoen, B., Demeulemeester, E., Beliën, J. (2010). Operating room planning and scheduling: A literature review, European journal of operational research, 201(3), 921-932.

[4] VanBerkel, P.T., Blake, J.T. (2007). A comprehensive simulation for wait time reduction and capacity planning applied in general surgery, Health care management Science, 10(4), 373-385.

[5] Favaretto, F. (2018). Management of Queues Assisted by Two Servers With Different Assistance Rates, Sistemas \& Gestao, 13(1), 2-9. 
[6] Paradi, J.C., Zhu, H. (2013). A survey on bank branch efficiency and performance research with data envelopment analysis, Omega, 41(1), 61-79.

[7] Banks, J., Carson II, J., Nelson, B., Nicol, D. Discrete-event system simulation, $5^{\text {th }}$ Edition, Prentice Hall, USA, 2009.

[8] Banks, J. Handbook of simulation: principles, methodology, advances, applications, and practice. John Wiley \& Sons, 1998.

[9] Sargent, R.G. (2010). Verification and validation of simulation models," In Proceedings of the 2010 IEEE winter simulation conference, Baltimore, MD, USA, 166-183.

[10] Sargent, R.G. (2013). Verification and validation of simulation models, Journal of simulation, 7(1), $12-24$

[11] Montevechi, J., Leal, F., de Pinho, A., da Silva Costa, R., de Oliveira, M., da Silva, A. (2010). Conceptual modeling in simulation projects by mean adapted IDEF: an application in a Brazilian tech company," In Proceedings of the 2010 IEEE winter simulation conference, Baltimore, MD, USA, 1624-1635.

[12] Gibbons, J.D., Olkin, I., Sobel, M. (1999). Selecting and Ordering Populations: A New Statistical Methodology, $2^{\text {nd }}$ Edition, SIAM, USA, 1999. 